\title{
Application of polymeric nanoparticles in food sector
}

\begin{abstract}
Nanotechnology presents opportunities to create new and better products. Nano technology has huge impact in many applications including food industry. Product of nanotechnology, such as polymeric nanoparticle, can be utilized to improve food quality by extending food shelf life, increase food safety, lower the cost and enhance the nutritional benefits. This chapter provides an overview of the properties of polymeric nanoparticle, preparation techniques, as well as the role polymeric nano-particles in the food industry.
\end{abstract}

